\title{
THE DIFFEOMORPHISM GROUP OF A LIE FOLIATION
}

\author{
G. HECTOR, E. MACÍAS-VIRGÓS, AND A. SOTELO-ARMESTO
}

\begin{abstract}
We explicitly compute the diffeomorphism group of several types of linear foliations (with dense leaves) on the torus $T^{n}, n \geq$ 2 , namely codimension one foliations, flows, and the so-called nonquadratic foliations. We show in particular that non-quadratic foliations are rigid, in the sense that they do not admit transverse diffeomorphisms other than $\pm \mathrm{id}$ and translations. The computation is an application of a general formula that we prove for the diffeomorphism group of any Lie foliation with dense leaves on a compact manifold. Our results generalize those of P. Donato and P. Iglesias for $T^{2}, \mathrm{P}$. Iglesias and G. Lachaud for codimension one foliations on $T^{n}, n \geq 2$, and B. Herrera for transcendent foliations. The theoretical setting of the paper is that of J. M. Souriau's diffeological spaces.
\end{abstract}

\section{INTRODUCTION}

J. M. Souriau's diffeological spaces [18] are a fruitful generalization of manifolds. In that setting one has natural constructions for subspaces, quotient spaces and spaces of maps. In particular, the factor space $G / \Gamma$ of the simply connected Lie group $G$ by any totally disconnected subgroup $\Gamma$ can be endowed with a diffeology, and its group of diffeomorphisms $\operatorname{Diff}(G / \Gamma)$ is a diffeological group - the generalization of the notion of Lie group. When $\Gamma$ is dense in $G$, we prove that the group $\operatorname{Diff}(G / \Gamma)$ is isomorphic to $\left(\operatorname{Aut}_{\Gamma}(G) \ltimes G\right) / \Gamma$. This formula will be our main tool.

For a $G$-Lie foliation [5, 6, 16] with holonomy $\Gamma$ on the compact manifold $M$, the space of leaves $M / \mathcal{F}$ turns out to be diffeomorphic (as a diffeological space) to $G / \Gamma$, hence the group $\operatorname{Diff}(M / \mathcal{F})$ of transverse diffeomorphisms can be computed by means of the formula above (when the leaves are dense). An important particular case (with $G=\mathbb{R}^{n}$ ) is that of linear foliations on the torus $T^{n}$.

In this paper we explicitly compute the diffeomorphism group $\operatorname{Diff}(M / \mathcal{F})$ for several types of linear foliations (with dense leaves) on the torus $T^{n}$, $n \geq 2$, namely codimension one foliations, flows, and the so-called nonquadratic foliations. We show in particular that non-quadratic foliations are rigid, in the sense that they do not admit transverse diffeomorphisms

2000 Mathematics Subject Classification. 57R30, 22E65, 58D05, 58B25.

Key words and phrases. diffeological space, diffeomorphism group, Lie foliation, linear flow.

Second author partially supported by FEDER and Research Project MTM2008-05861 MICINN Spain. 
other than \pm id and translations. Our results generalize those of P. Donato and P. Iglesias [2] for $T^{2}$, P. Iglesias and G. Lachaud [12] for codimension one foliations on $T^{n}, n \geq 2$, and B. Herrera [9, 10] for transcendent foliations.

\section{Diffeological Spaces}

The notion of diffeological space is due to J. M. Souriau [18]. We shall briefly review the basic constructions on the category of diffeological spaces that we need along this paper. Proofs are left to the reader, see also [8, 7, 13].

Let $M$ be a set. Any set map $\alpha: U \subset \mathbb{R}^{n} \rightarrow M$ defined on an open set $U$ of some $\mathbb{R}^{n}, n \geq 0$, will be called a plot on $M$. The name plot is chosen instead of chart to avoid some confusion with the usual notion of chart in a manifold. When possible, a plot $\alpha$ with domain $U$ will be simply denoted by $\alpha_{U}$.

Definition 2.1. A diffeology of class $\mathcal{C}^{\infty}$ on the set $M$ is any collection $\mathcal{P}$ of plots $\alpha: U_{\alpha} \subset \mathbb{R}^{n_{\alpha}} \rightarrow M, n_{\alpha} \geq 0$, verifying the following axioms:

(1) Any constant map $c: \mathbb{R}^{n} \rightarrow M, n \geq 0$, belongs to $\mathcal{P}$;

(2) Let $\alpha \in \mathcal{P}$ be defined on $U \subset \mathbb{R}^{n}$ and let $h: V \subset \mathbb{R}^{m} \rightarrow U \subset \mathbb{R}^{n}$ be any $\mathcal{C}^{\infty}$ map; then $\alpha \circ h \in \mathcal{P}$;

(3) Let $\alpha: U \subset \mathbb{R}^{n} \rightarrow M$ be a plot. If any $t \in U$ has a neighbourhood $U_{t}$ such that $\alpha_{\mid U_{t}}$ belongs to $\mathcal{P}$ then $\alpha \in \mathcal{P}$.

Usually, a diffeology $\mathcal{P}$ on the set $M$ is defined by means of a generating set, that is by giving any set $\mathcal{G}$ of plots (which is implicitly supposed to contain all constant maps) and taking the least diffeology containing it. Explicitly, the diffeology $\langle\mathcal{G}\rangle$ generated by $\mathcal{G}$ is the set of plots $\alpha: U \rightarrow M$ such that any point $t \in U$ has a neighbourhood $U_{t}$ where $\alpha$ can be written as $\gamma \circ h$ for some $\mathcal{C}^{\infty}$ map $h$ and some $\gamma \in \mathcal{G}$.

Example 2.2. A finite dimensional manifold $M$ is endowed with the diffeology generated by the charts $U \subset \mathbb{R}^{n} \rightarrow M, n=\operatorname{dim} M$, of any atlas.

2.1. Basic constructions. A map $F:(M, \mathcal{P}) \rightarrow(N, \mathcal{Q})$ between diffeological spaces is differentiable if $F \circ \alpha \in \mathcal{Q}$ for all $\alpha \in \mathcal{P}$. A diffeomorphism is a differentiable map with a differentiable inverse.

Let $(M, \mathcal{P})$ be a diffeological space and $F: M \rightarrow N$ a map of sets. The final diffeology $F_{\star} \mathcal{P}$ on $N$ is that generated by the plots $F \circ \alpha, \alpha \in \mathcal{P}$. A particular case is the quotient diffeology associated to an equivalence relation on $M$.

Analogously, let $(N, \mathcal{Q})$ be a diffeological space and $F: M \rightarrow N$ a map of sets. The initial diffeology $F^{\star} \mathcal{Q}$ on $M$ is that generated by the plots $\alpha$ in $M$ such that $F \circ \alpha \in \mathcal{Q}$. A particular case is the induced diffeology on any subset $M \subset N$.

Both constructions verify the usual universal properties.

Let $(M, \mathcal{P}),(N, \mathcal{Q})$ be two diffeological spaces. We can endow the cartesian product $M \times N$ with the product diffeology $\mathcal{P} \times \mathcal{Q}$ generated by the plots $\alpha \times \beta, \alpha \in \mathcal{P}, \beta \in \mathcal{Q}$. 
Finally, let $\mathcal{D}(M, N)$ be the space of differentiable maps between two diffeological spaces $(M, \mathcal{P})$ and $(N, \mathcal{Q})$. We define the functional diffeology on it by taking as a generating set all plots $\alpha: U \rightarrow \mathcal{D}(M, N)$ such that the associated map $\widehat{\alpha}: U \times M \rightarrow N$ given by $\widehat{\alpha}(t, x)=\alpha(t)(x)$ is differentiable.

\subsection{Diffeological groups.}

Definition 2.3. A diffeological group is a diffeological space $(G, \mathcal{P})$ endowed with a group structure such that the division map $\delta: G \times G \rightarrow G, \delta(x, y)=$ $x y^{-1}$, is differentiable.

A typical example of diffeological group is the diffeomorphism group of a finite dimensional manifold $M$, endowed with the diffeology induced by $\mathcal{D}(M, M)$ [7]. In [8] (see also Prop. 4.2) it is proven that the diffeomorphism group of the space of leaves of a Lie foliation is a diffeological group too. The aim of the present paper is to compute it.

\section{HomogeneOUS SPACES AND COVERING MAPS}

Let $G$ be a connected and simply connected Lie group, $\Gamma \subset G$ an arbitrary totally disconnected subgroup. While it is possible to develop a general theory of fibre bundles and covering spaces in the diffeological setting [11, we shall directly prove some lifting properties for the quotient map $\pi: G \rightarrow$ $G / \Gamma$. These lifting properties will be essential later.

3.1. Lifting of diffeomorphisms. The factor space $G / \Gamma$ is endowed with the quotient diffeology, that is the collection of plots $\alpha: U \rightarrow G / \Gamma$ which locally lift through $\pi$ to a smooth map $U \rightarrow G$.

Proposition 3.1. Any differentiable map (in the diffeological sense) $\bar{\varphi}: G / \Gamma \rightarrow$ $G / \Gamma$ has a smooth lift $\varphi: G \rightarrow G$, that is a $\mathcal{C}^{\infty}$ map such that $\pi \varphi=\bar{\varphi} \pi$.

Proof. By definition of quotient diffeology, the result is locally true, that is for any $x \in G$ there exists an open neighbourhood $U_{x}$ and a smooth map $\varphi_{x}: U_{x} \rightarrow G$ such that $\pi \circ \varphi_{x}=\bar{\varphi} \circ \pi$ on $U_{x}$. We can suppose that $U_{x}$ is a connected open set.

Now we define an integrable distribution $\mathcal{D}$ on $G \times G$ in the following way. Since an arbitrary point $(x, g) \in G \times G$ can be written as $\left(x, \varphi_{x}(x) h\right)$ for some $h \in G$, let

$$
\mathcal{D}_{(x, g)}=\left\{\left(v,\left(R_{h} \circ \varphi_{x}\right)_{* x}(v)\right): v \in T_{x} G\right\} \subset T_{(x, g)}(G \times G) .
$$

The distribution $\mathcal{D}$ is well defined because two local lifts differ by some translation. In fact, let $x, y \in G$ such that $U_{x} \cap U_{y} \neq \emptyset$. Then for any $z \in U_{x} \cap U_{y}$ and any connected neighbourhood $V_{z} \subset U_{x} \cap U_{y}$, the local lifts $\varphi_{x}, \varphi_{y}$ define the continuous map $\gamma: V_{z} \rightarrow \Gamma$ given by $\gamma(t)=\varphi_{x}(t)^{-1} \varphi_{y}(t)$. Since $V_{z}$ is connected and the set $\Gamma$ is totally disconnected, the map $\gamma$ must be constant, hence $\varphi_{y}=R_{\gamma} \circ \varphi_{x}$.

Moreover $\mathcal{D}$ has constant rank, and it is integrable, the integral submanifolds being translations of the graphs of the local lifts. 
Let us choose some point $x_{0} \in G$ such that $\left[x_{0}\right]=\bar{\varphi}([e])$, and let $\widetilde{G}$ be the maximal integral submanifold passing through $\left(e, x_{0}\right)$. We shall prove in the next Lemma 3.2 that the projection of $\widetilde{G} \subset G \times G$ onto the first factor is a covering map. Since $G$ is simply connected, it follows that $\widetilde{G}$ is the graph of a global lift.

Lemma 3.2. The projection $p_{1}: \widetilde{G} \subset G \times G \rightarrow G$ is a covering map.

Proof. Clearly $p_{1}$ is a differentiable submersion, hence an open map, so $p_{1}(\widetilde{G})$ is an open subspace of $G$. Let us prove that it is closed too; this will show that the map $p_{1}: \widetilde{G} \rightarrow G$ is onto, because the Lie group $G$ is connected.

Suppose $x \in G$ is in the closure of $p_{1}(\widetilde{G})$, and let $U_{x}$ be a connected open neighbourhood where the local lift $\varphi_{x}$ is defined. Let $y \in U_{x} \cap p_{1}(\widetilde{G})$, then $\left(y, \varphi_{x}(y) h\right) \in \widetilde{G}$ for some $h \in G$. This implies that the graph of $R_{h} \circ \varphi_{x}$, which is an integral submanifold of $\mathcal{D}$, is contained in $\widetilde{G}$. Hence $x \in p_{1}(\widetilde{G})$.

It remains to prove that any $x \in G$ has a neighbourhood $U_{x}$ such that $\left(p_{1}\right)^{-1}\left(U_{x}\right)$ is a disjoint union of open sets, each one homeomorphic to $U_{x}$ by $p_{1}$. It is clear that we can restrict ourselves to the case $x=e$. Let $\varphi_{e}: U_{e} \rightarrow G$ be a connected local lift of $\bar{\varphi}$. We can suppose that $\varphi_{e}(e)=x_{0}$. Let $\widetilde{U_{e}}$ be its graph. Then $\widetilde{U_{e}}$ is an open subset of $\widetilde{G}$, containing $\left(e, x_{0}\right)$, with $p_{1}\left(\widetilde{U_{e}}\right)=U_{e}$. Let $I$ be the non-empty set

$$
I=\left\{\gamma \in \Gamma:\left(e, x_{0} \gamma\right) \in \widetilde{G}\right\} .
$$

Then $\left(p_{1}\right)^{-1}\left(U_{e}\right)$ is the disjoint union of the sets $R_{\gamma}\left(\widetilde{U_{e}}\right), \gamma \in I$.

Corollary 3.3. Any diffeomorphism of $G / \Gamma$ can be lifted to a diffeomorphism of $G$.

Proof. In the proof of Theorem 3.1 , the integral manifold $\widetilde{G}$ passing through a given point is unique, hence the lift of the map $\bar{\varphi}$ is unique once one has selected a representative of $\bar{\varphi}([e])$. Then the appropriate lifts of $\bar{\varphi}$ and $(\bar{\varphi})^{-1}$ are inverse maps.

Analogous arguments give the following result.

Corollary 3.4. Let $U$ be a connected simply connected open subset of $\mathbb{R}^{n}$, $n \geq 0$. Any differentiable map (resp. diffeomorphism) $U \times G / \Gamma \rightarrow U \times G / \Gamma$ can be lifted to a $\mathcal{C}^{\infty}$ map (resp. diffeomorphism) $U \times G \rightarrow U \times G$.

\section{The DIFFEOMORPHism GRoup}

Several results in this section were previously announced by two of the authors in [8]. For the sake of completeness, we shall give complete proofs.

Let $G / \Gamma$ be the factor space (endowed with the quotient diffeology) of the connected simply connected Lie group $G$ by the totally disconnected subgroup $\Gamma$. Let $\operatorname{Diff}(G / \Gamma)$ be the diffeomorphism group of $G / \Gamma$, with the diffeology induced by the functional diffeology of $\mathcal{D}(G / \Gamma, G / \Gamma)$. 
Lemma 4.1. Any plot in $\operatorname{Diff}(G / \Gamma)$ with simply connected domain can be lifted to some plot in $\operatorname{Diff}(G)$.

Proof. Let $\alpha: U \subset \mathbb{R}^{n} \rightarrow \operatorname{Diff}(G / \Gamma) \subset \mathcal{D}(G / \Gamma, G / \Gamma)$ be a plot in the functional diffeology, that is such that the map

$$
\widehat{F}: U \times G / \Gamma \rightarrow U \times G / \Gamma, \quad \widehat{F}(t,[g])=(t, \alpha(t)([g])),
$$

is differentiable. Take (Corollary 3.4) a lift $F: U \times G \rightarrow U \times G$. Then $F(t, g)=(t, \xi(t, g))$, where $[\xi(t, g)]=\alpha(t)([g])$. For each $t \in U$, lift $\alpha(t)^{-1}$ to some diffeomorphism $\lambda_{t}$ of the Lie group $G$. Since $\left[\left(\lambda_{t} \circ \xi\right)(t, g)\right]=[g]$ for all $g \in G$, we have $\lambda_{t} \circ \xi(t,-)=R_{\gamma}$ for some $\gamma \in \Gamma$, because the Lie group $G$ is connected and the subgroup $\Gamma$ is totally disconnected. Analogously $\xi(t,-) \circ \lambda_{t}=R_{\mu}$ for some $\mu \in \Gamma$. This proves that $\xi(t,-)$ is a diffeomorphism of $G$, so $\xi: U \rightarrow \operatorname{Diff}(G)$ is the desired lift.

Theorem $4.2([8])$. $\operatorname{Diff}(G / \Gamma)$ is a diffeological group, with the diffeology induced by $\mathcal{D}(G / \Gamma, G / \Gamma)$.

Proof. The differentiability of the composition follows from the very definitions (for the diffeomorphism group of any diffeological space). The crucial point is to prove that the inversion map $I: \operatorname{Diff}(G / \Gamma) \rightarrow \operatorname{Diff}(G / \Gamma)$ is differentiable.

Let $\alpha_{U}$ be a plot in $\operatorname{Diff}(G / \Gamma)$. We can suppose that the domain $U$ is simply connected. By applying the inverse function theorem to the manifold $U \times G$ we conclude that the map $F$ in Lemma 4.1 is a diffeomorphism. But $F^{-1}$ is a lifting of the map $(t, g) \in U \times G / \Gamma \mapsto\left(t, \alpha(t)^{-1}([g])\right) \in U \times G / \Gamma$, which proves that $I \circ \alpha$ is a plot in the functional diffeology.

4.1. An explicit formula. Let us suppose that the subgroup $\Gamma$ is dense in G.

We denote $\operatorname{Aut}_{\Gamma}(G) \subset \operatorname{Aut}(G)$ the group of automorphisms of the Lie group $G$ which preserve the subgroup $\Gamma$. Let us consider the semidirect product $\operatorname{Aut}_{\Gamma}(G) \ltimes G$, where the product is given by $\left(\theta_{1}, g_{1}\right)\left(\theta_{2}, g_{2}\right)=$ $\left(\theta_{1} \theta_{2}, g_{1} \theta_{1}\left(g_{2}\right)\right)$. The group $\Gamma$ can be identified with a subgroup of that semidirect product by means of the map $i(\gamma)=\left(i_{\gamma}, \gamma^{-1}\right)$, where $i_{\gamma}$ is the inner automorphism $i_{\gamma}(g)=\gamma g \gamma^{-1}$. This subgroup is invariant, because

$$
(\theta, g)\left(i_{\gamma}, \gamma^{-1}\right)(\theta, g)^{-1}=\left(i_{\theta(\gamma)}, \theta(\gamma)^{-1}\right)
$$

Theorem 4.3. Let $G$ be a connected simply connected Lie group, $\Gamma \subset G$ a totally disconnected dense subgroup. Then $\operatorname{Diff}(G / \Gamma)$ is isomorphic to the quotient group $\left(\operatorname{Aut}_{\Gamma}(G) \ltimes G\right) / \Gamma$.

This formula will be our main tool. The proof (sketched in [8]) will be divided into several Lemmas.

Lemma 4.4. Let $\varphi$ be any diffeomorphism of $G$ which induces a diffeomorphism on $G / \Gamma$. Let us define $\theta_{\varphi}=L_{\varphi(e)}^{-1} \circ \varphi$. Then $\theta_{\varphi} \in \operatorname{Aut}_{\Gamma}(G)$. Moreover $\theta_{\varphi \circ \psi}=\theta_{\varphi} \circ \theta_{\psi}$. 
Proof. We have $\varphi(g)=\varphi(e) \theta_{\varphi}(g)$, for all $g \in G$. But in fact $\varphi(g \gamma)=$ $\varphi(g) \theta_{\varphi}(\gamma)$ for all $\gamma \in \Gamma$, because for any fixed $\gamma$ the continuous map $\varphi(g)^{-1} \varphi(g \gamma)$ defined on $G$ takes its values in the totally disconnected group $\Gamma$, hence it is constant. Moreover $\theta_{\varphi}(\gamma) \in \Gamma$.

Now, for $\gamma, \mu \in \Gamma$ we have

$$
\theta_{\varphi}(\mu \gamma)=\varphi(e)^{-1} \varphi(\mu \gamma)=\varphi(e)^{-1} \varphi(\mu) \theta_{\varphi}(\gamma)=\theta_{\varphi}(\mu) \theta_{\varphi}(\gamma)
$$

hence $\theta_{\varphi}$ is a group morphism when restricted to $\Gamma$. Since the subgroup $\Gamma \subset G$ is dense, it follows that $\theta_{\varphi}$ is an automorphism of $G$.

Finally, for two diffeomorphisms $\varphi, \psi$ we have

$$
\varphi \psi=L_{\varphi(e)} \theta_{\varphi} L_{\psi(e)} \theta_{\psi}=L_{\varphi(e)} L_{\theta_{\varphi} \psi(e)} \theta_{\varphi} \theta_{\psi}=L_{\varphi \psi(e)} \theta_{\varphi} \theta_{\psi}
$$

which proves $\theta_{\varphi \psi}=\theta_{\varphi} \theta_{\psi}$.

Remark 4.5. Clearly, $\theta_{\varphi}=$ id if and only if $\varphi$ is a (left) translation by an element of $G$. On the other hand, right translations by elements of $\Gamma$ induce the identity on $G / \Gamma$.

We know from Theorem 3.1 that any diffeomorphism $\bar{\varphi}$ of $G / \Gamma$ can be lifted to some diffeomorphism $\varphi$ of $G$.

Lemma 4.6. The map $\Phi: \operatorname{Diff}(G / \Gamma) \rightarrow\left(\operatorname{Aut}_{\Gamma}(G) \ltimes G\right) / \Gamma$ given by

$$
\Phi(\bar{\varphi})=\left[\left(\theta_{\varphi}, \varphi(e)\right)\right]
$$

is an isomorphism of groups.

Proof. That the map $\Phi$ is well defined follows from the fact that if $\varphi, \psi$ are two different lifts of $\bar{\varphi}$, then $\theta_{\psi}=i_{\gamma} \circ \theta_{\varphi}$, where $i_{\gamma}$ is the inner automorphism with $\gamma=\psi(e)^{-1} \varphi(e)$, hence we have

$$
\left(\theta_{\psi}, \psi(e)\right)=\left(i_{\gamma}, \gamma^{-1}\right)\left(\theta_{\varphi}, \varphi(e)\right)
$$

Let us prove that $\Phi$ is a bijective map. First it is injective, because if $\bar{\varphi}$ goes into the identity, then $\theta_{\varphi}=i_{\gamma}$ and $\varphi(e)=\gamma^{-1}$, for some $\gamma \in \Gamma$, which implies $\varphi=R_{\gamma}^{-1}$, that is $\bar{\varphi}$ is the identity of $G / \Gamma$. On the other hand, $\Phi$ is onto because for any pair $(\theta, g) \in \operatorname{Aut}_{\Gamma}(G) \ltimes G$ we can take the diffeomorphism $\varphi=L_{g} \circ \theta$.

That $\Phi$ is a morphism of groups follows from Lemma 4.4.

Lemma 4.7. $\Phi$ is a diffeomorphism of diffeological spaces.

Proof. From the definition of induced diffeology (Subsection 2.1) it turns out that any subgroup of a diffeological group is a diffeological group. This applies to $\operatorname{Aut}_{\Gamma}(G) \subset \operatorname{Diff}(G)$, and also to the subgroup $\operatorname{Diff}(G: \Gamma)$ of diffeomorphisms of $G$ which induce a diffeomorphism on $G / \Gamma$. We have the 
following diagram of exact sequences of diffeological groups:

$\begin{array}{cccccc}Z(\Gamma) & \rightarrow & G & & G & G / Z(\Gamma) \\ \downarrow & & L \downarrow & & L \downarrow \\ \Gamma & & R & \operatorname{Diff}(G: \Gamma) & \rightarrow & \operatorname{Diff}(G / \Gamma) \\ i \downarrow & & \theta \downarrow & & & \downarrow \\ i(\Gamma) & \rightarrow & \operatorname{Aut}_{\Gamma}(G) & \rightarrow & \operatorname{Aut}_{\Gamma}(G) / i(\Gamma)\end{array}$

where $i(\gamma)$ is an inner automorphism, $R(\gamma)$ is a right translation, and $L(g)$ is a left translation. Then the differentiability of $\Phi$ and $\Phi^{-1}$ is an easy consequence of Lemma 4.1 and the diagram above.

This completes the proof of Theorem 4.3.

\section{LiE FOLIATIONS}

Lie foliations play a central role in the study of transversely riemannian foliations [16]. Let $\mathfrak{g}$ be a Lie algebra of dimension $n$. A $\mathfrak{g}$-Lie foliation on the manifold $M$ is defined as the kernel of a non-degenerate 1-form $\omega$ with values in $\mathfrak{g}$, such that $d \omega=(-1 / 2)[\omega, \omega]$. Once a basis $X_{1}, \ldots, X_{n}$ of $\mathfrak{g}$ with structural constants $c_{i j}^{k}$ has been fixed, this is equivalent to having $n$ independent real 1-forms $\omega_{1}, \ldots, \omega_{n}$ on $M$ such that $d \omega_{k}=\sum c_{i j}^{k} \omega_{i} \wedge \omega_{j}$.

When the manifold $M$ is compact, it is well known [5, 15] that there exists a regular covering $p: \widetilde{M} \rightarrow M$ such that the lifted foliation $p^{\star} \mathcal{F}$ is a locally trivial bundle $D: \widetilde{M} \rightarrow G$ over the connected simply connected Lie group $G$ associated to $\mathfrak{g}$. By fixing base points $x_{0} \in M$ and $\widetilde{x}_{0} \in \widetilde{M}$ with $p\left(\widetilde{x}_{0}\right)=x_{0}$ and $D\left(\widetilde{x}_{0}\right)=e \in G$, one obtains a group morphism $h: \pi_{1}(M) \rightarrow G$ such that ker $h=\pi_{1}(\widetilde{M})$. Moreover $D$ is $h$-equivariant. The developing map $D$ and the holonomy morphism $h$ completely determine the foliation. The image $\Gamma$ of $h$ is called the holonomy group; it is dense in $G$ if and only if all the leaves of $\mathcal{F}$ are dense in $M$.

From the definitions above it is easy to prove that the space of leaves $M / \mathcal{F}$ (endowed with the quotient diffeology) is diffeomorphic to the factor space $G / \Gamma$.

Proposition 5.1. For any Lie foliation $\mathcal{F}$ on the manifold $M$, the groups $\operatorname{Diff}(M / \mathcal{F})$ and $\operatorname{Diff}(G / \Gamma)$ are isomorphic.

When $M$ is compact, the group $\Gamma=\operatorname{im} h$ is finitely generated, so we can apply our previous computation of the diffeomorphism group.

5.1. Linear foliations on the torus. A particular case of Lie foliation is that of a linear foliation on the torus. An arbitrary linear foliation $\mathcal{F}$ of dimension $m \geq 1$ and codimension $n \geq 1$ on the torus $M=T^{m+n}$ is determined by some linear subspace $V \subset \mathbb{R}^{m+n}$ of dimension $m$. The holonomy group $\Gamma$ is a finitely generated subgroup of $\mathbb{R}^{n}$, hence a free abelian group of rank $k$. Let $V^{\perp}$ be the orthogonal subspace to $V$, and take arbitrary basis $v_{1}, \ldots, v_{m}$ of $V$ and $w_{1}, \ldots, w_{n}$ of $V^{\perp}$. Then the closed 1-form $\omega=$ $\left(d w_{1}, \ldots, d w_{n}\right)$ with values in $\mathbb{R}^{n}$ defines the foliation $\mathcal{F}$. 
The holonomy morphism $\pi_{1}\left(T^{m+n}\right)=\mathbb{Z}^{m+n} \rightarrow \mathbb{R}^{n}$ is obtained by computing the group of periods of the form $\omega$, then $\Gamma=D\left(\mathbb{Z}^{m+n}\right)$ if we take as a developping map the orthogonal projection $D: \mathbb{R}^{m+n} \rightarrow V^{\perp}=\mathbb{R}^{n}$. Note that in general $V \cap \mathbb{Z}^{m+n} \neq 0$, which means that the covering $\widetilde{M}$ of Section 5 is not the universal covering $\mathbb{R}^{m+n}$ but the intermediate covering corresponding to ker $h$.

Our general formula in 4.1 reduces to $\operatorname{Diff}\left(T^{m+n} / \mathcal{F}\right)=\operatorname{Aut}_{\Gamma}\left(\mathbb{R}^{n}\right) \times$ $\left(\mathbb{R}^{n} / \Gamma\right)$ because the group $G=\mathbb{R}^{n}$ is abelian.

Remark 5.2. In [3, 4] A. El Kacimi and A. Tihami computed the bigraded cohomology of linear foliations on tori.

5.2. Duality. We can establish some kind of duality between the foliations defined by $V$ and $V^{\perp}$. This idea will play an important role in our paper. First, in order to compute the group of transverse diffeomorphisms we shall suppose that the leaves of the foliation are simply connected (planes). If this is not the case, we just need to reduce the dimension of the ambient torus. This condition is equivalent to $V \cap \mathbb{Z}^{m+n}=0$, which means that the rank $k$ of $\Gamma$ equals $m+n$, because $D: \mathbb{Z}^{m+n} \subset \mathbb{R}^{m+n} \rightarrow \Gamma \subset \mathbb{R}^{n}$ is an isomorphism.

Lemma 5.3. A finitely generated subgroup $\Gamma$ of $\mathbb{R}^{p}$ is dense if and only if $f(\Gamma)$ is dense in $\mathbb{R}$ for any non-degenerate linear form $f: \mathbb{R}^{p} \rightarrow \mathbb{R}$.

We leave the proof to the reader.

Proposition 5.4. $V \cap \mathbb{Z}^{m+n}=0$ if and only if the orthogonal foliation $\mathcal{F}^{\perp}$ determined by $V^{\perp}$ has dense leaves.

Proof. Let $D^{\perp}: \mathbb{R}^{m+n} \rightarrow V=\mathbb{R}^{m}$ be the orthogonal projection onto $V$. The holonomy group of $\mathcal{F}^{\perp}$ is $\Gamma^{*}=D^{\perp}\left(\mathbb{Z}^{m+n}\right)$. If $\Gamma^{*}$ is not dense in $\mathbb{R}^{m}$, then by Lemma 5.3 there exists some non trivial linear map $f: \mathbb{R}^{m} \rightarrow \mathbb{R}$ such that $f\left(\Gamma^{*}\right)$ is not dense in $\mathbb{R}$, hence it is a non-zero discrete subgroup. By multiplying by some scalar we can suppose that $f\left(\Gamma^{*}\right)=\mathbb{Z}$. Composing $f$ with $D^{\perp}$ then gives a linear map $\varphi: \mathbb{R}^{m+n} \rightarrow \mathbb{R}$ such that $\varphi\left(V^{\perp}\right)=0$ and $\varphi\left(\mathbb{Z}^{m+n}\right)=\mathbb{Z}$. Let $v \in V, v \neq 0$, such that $\varphi=\langle v,-\rangle$. Then $\left\langle v, \mathbb{Z}^{m+n}\right\rangle \subset \mathbb{Z}$ means that $v$ has integer coordinates with respect to the canonical basis of $\mathbb{R}^{m+n}$, that is $v \in V \cap \mathbb{Z}^{m+n} \neq 0$.

The reciprocal is completely analogous.

Corollary 5.5. Suppose that $\mathcal{F}$ has simply connected dense leaves. Then so has $\mathcal{F}^{\perp}$, and the groups $\operatorname{Aut}_{\Gamma}\left(\mathbb{R}^{n}\right)$ and $\operatorname{Aut}_{\Gamma^{*}}\left(\mathbb{R}^{m}\right)$ are isomorphic.

Proof. Computing $\operatorname{Aut}_{\Gamma}\left(\mathbb{R}^{n}\right)$ is equivalent to finding the matrices $A \in P S L(m+$ $n, \mathbb{Z})$ such that $A(V)=V$. Clearly this is equivalent to the condition $A^{T}\left(V^{\perp}\right)=V^{\perp}$ when $V \cap \mathbb{Z}^{m+n}=0$ and $V^{\perp} \cap \mathbb{Z}^{m+n}=0$.

Later in Section 7 we shall use the fact that this duality always occurs when $\mathcal{F}$ is a dense flow.

Remark 5.6. All along the following sections we shall consider the following Moebius action of $G L(k, \mathbb{Z})$ on the projective space $P \mathbb{R}^{k-1}$. Let $A=\left(a_{i j}\right)$ 
be an invertible $k \times k$ integer matrix (hence $\operatorname{det} A= \pm 1$ ), and let $v=$ $\left(v_{2}, \ldots, v_{k}\right) \in \mathbb{R}^{k-1}$. Then $A \cdot v=w=\left(w_{2}, \ldots, w_{k}\right)$ is given by

$$
w_{j}=\frac{a_{1 j}+a_{2 j} v_{2}+\cdots+a_{n j} v_{k}}{a_{11}+a_{21} v_{2}+\cdots+a_{n 1} v_{k}}, \quad 2 \leq j \leq k .
$$

We shall say that the vectors $v, w$ are $G L(k, \mathbb{Z})$-related.

\section{Codimension one linear foliations on the torus}

Remark 6.1. Theorem 6.5 in this section was obtained long before us by P. Iglesias and G. Lachaud in 12. We are indebted to Professor Iglesias for pointing us the existence of their article, which we were unaware when we wrote our paper.

6.1. Classification. Let $\mathcal{F}$ be a codimension one linear foliation with dense leaves on the torus $T^{m+1}=\mathbb{R}^{m+1} / \mathbb{Z}^{m+1}$. It is given by a closed 1 -form $\omega=d y-\xi_{1} d x_{1}-\cdots-\xi_{n} d x_{m}$, where at least one of the real numbers $\xi_{1}, \ldots, \xi_{m}$ is not rational. The holonomy group $\Gamma=\left\langle 1, \xi_{1}, \ldots, \xi_{m}\right\rangle$ is a dense finitely generated subgroup of $\mathbb{R}$, hence a free abelian group of rank $k$.

Our first proposition is a classification of the spaces of leaves, which generalizes the corresponding result of $\mathrm{P}$. Donato and P. Iglesias for $T^{2}$ [2].

Proposition 6.2. Let $\mathcal{F}^{\prime}$ be another codimension one linear foliation with dense leaves on $T^{m+1}$, and let $\Gamma^{\prime} \subset \mathbb{R}$ be its holonomy group. Then the spaces of leaves $\mathbb{R} / \Gamma$ and $\mathbb{R} / \Gamma^{\prime}$ are diffeomorphic if and only if $\Gamma$ and $\Gamma^{\prime}$ have equal ranks, and for any respective basis $\Gamma=\left\langle\alpha_{1}, \ldots, \alpha_{k}\right\rangle, \Gamma^{\prime}=\left\langle\beta_{1}, \ldots, \beta_{k}\right\rangle$, $k \leq m+1$, the vectors $\alpha_{1}^{-1}\left(\alpha_{2}, \ldots, \alpha_{k}\right)$ and $\beta_{1}^{-1}\left(\beta_{2}, \ldots, \beta_{k}\right)$ are $G L(k, \mathbb{Z})$ related.

In other words, there is some integer matrix $A=\left(a_{i j}\right) \in G L(k, \mathbb{Z})$ such that

$$
\beta_{1}^{-1} \beta_{j}=\left(a_{1 j} \alpha_{1}+\cdots+a_{k j} \alpha_{k}\right) / d, \quad 2 \leq j \leq k,
$$

where $d=a_{11} \alpha_{1}+\cdots+a_{k 1} \alpha_{k}$.

Proof. Suppose that the spaces of leaves $\mathbb{R} / \Gamma$ and $\mathbb{R} / \Gamma^{\prime}$ are diffeomorphic as diffeological spaces. By the lifting property proved in Theorem 3.1, there is a diffeomorphism $\varphi: \mathbb{R} \rightarrow \mathbb{R}$ sending $\Gamma^{\prime}$ into $\Gamma$. Then $\Gamma$ and $\Gamma^{\prime}$ have equal ranks.

Moreover, the associated linear automorphism $\theta=-\varphi(0)+\varphi$ of $\mathbb{R}$ (see Lemma 4.4) must be a homothety $\theta(t)=\lambda t$ for some $\lambda \in \mathbb{R}, \lambda \neq 0$. Since $\theta$ restricted to $\Gamma^{\prime}$ is an isomorphism into $\Gamma$, we have

$$
\lambda \beta_{j}=a_{1 j} \alpha_{1}+\cdots+a_{m j} \alpha_{k}, \quad 1 \leq j \leq k,
$$

with $a_{i j} \in \mathbb{Z}$. If we take $d=\lambda \beta_{1}$ the result follows. The converse is immediate. 
Remark 6.3. Notice that if $\Gamma, \Gamma^{\prime}$ have equal rank $k$, then $\mathbb{R} / \Gamma$ and $\mathbb{R} / \Gamma^{\prime}$ are always isomorphic as groups. In fact, since $\mathbb{R}$ is a $\mathbb{Q}$-vector space, take

$$
V=\left\langle\alpha_{1}, \ldots, \alpha_{k}, \beta_{1}, \ldots, \beta_{k}\right\rangle_{\mathbb{Q}}
$$

which is a $\mathbb{Q}$-vector space of finite dimension $\geq k$, and write $\mathbb{R}=V \oplus W$ (by Zorn's lemma). Since $\left\{\alpha_{1}, \ldots, \alpha_{k}\right\}$ and $\left\{\beta_{1}, \ldots, \beta_{k}\right\}$ are linearly independent sets over $\mathbb{Q}$, there exists $f: V \rightarrow V$ such that $f\left(\alpha_{i}\right)=\beta_{i}, 1 \leq i \leq k$, and we can extend it to $\mathbb{R}$ by putting $f=$ id on $W$.

Corollary 6.4 ([2]). For the torus $T^{2}$, two irrational linear flows $\mathcal{F}_{\alpha}, \mathcal{F}_{\beta}$ with holonomy groups $\Gamma_{\alpha}=\langle 1, \alpha\rangle, \Gamma_{\beta}=\langle 1, \beta\rangle$ have diffeomorphic (and isomorphic) spaces of leaves if and only if there is some integer matrix

$$
A=\left(\begin{array}{ll}
a & c \\
b & d
\end{array}\right)
$$

such that

$$
\beta=\frac{c+d \alpha}{a+b \alpha}
$$

\subsection{The group of diffeomorphisms.}

Theorem 6.5 ([12]). For any codimension one linear foliation on the torus $T^{m+1}$ with dense holonomy group $\Gamma$, the group $\operatorname{Aut}_{\Gamma}(R)$ is isomorphic to $\mathbb{Z}_{2} \times \mathbb{Z} \times \stackrel{r)}{\cdots} \times \mathbb{Z}, r \leq m$.

A very elegant proof is given in the paper [12] from Iglesias and Lachaud. A more constructive proof has been done by the third author of the present paper in [17] as an application of the general formula in subsection 4.1. We summarize it.

Proof. Let $B=\left\{\alpha_{1}, \ldots, \alpha_{k}\right\}$ be a basis of the free abelian group $\Gamma$. We can suppose without loose of generality that $\alpha_{1}=1$. We have to consider three different cases.

(1) If the basis $B$ is an algebraic basis over the rationals of some extension $\mathbb{Q}(\beta)$ of finite degree over $Q$, let $A$ be the integral closure of $\mathbb{Q}(\beta)$ over $\mathbb{Z}[19]$. Since $\Gamma$ is a $\mathbb{Z}$-module of finite type with the same rank that $A$, we must have $\Gamma \subset(1 / q) A$ for some positive integer $q$. It follows that $\operatorname{Aut}_{\Gamma}(\mathbb{R}) \subset \operatorname{Aut}_{A}(\mathbb{R})=U(A)$. Hence, by Dirichlet's units theorem, $\operatorname{Aut}_{\Gamma}(\mathbb{R})=\mathbb{Z}_{2} \times \mathbb{Z} \times \cdots \times \mathbb{Z}$, with $0 \leq r \leq s+t-1$, where $t$ is the number of real roots of the minimal polynomial of $\beta$ over $\mathbb{Q}$, and $2 s$ is the number of complex roots. The torsion subgroup is $\mathbb{Z}_{2}$ because \pm 1 are the only real roots of the unit.

(2) If the basis $B$ is not the basis of an algebraic extension, but all its elements are algebraic, since $1 \in B$, any $\lambda \in \mathbb{R}-\{0\}$ inducing an automorphism of $\Gamma$ must verify $\lambda^{p} \in \Gamma$ for all $p \in \mathbb{Z}$. Since the elements of $B$ are algebraic and in a finite number, we can consider the minimal algebraic extension $\mathbb{Q}(\beta)$ of finite degree over $\mathbb{Q}$ containing $B$. Then $\operatorname{Aut}_{\Gamma}(\mathbb{R}) \subset U(A)$ as before. 
(3) Finally, if the basis $B$ is not the basis of any algebraic extension, and contains transcendent elements, let $\Gamma_{A} \subset \Gamma$ be the subgroup of elements of $\Gamma$ which are algebraic numbers over $\mathbb{Q}$. Choose a basis $B_{A}$ of $\Gamma_{A}$ and complete it to a basis $B$ of $\Gamma$. Let $B_{T}=B-B_{A}$ the trascendent part of $B$. Let $\lambda \in \operatorname{Aut}_{\Gamma}(\mathbb{R})$. Again $0 \neq \lambda^{p} \in \Gamma=\langle B\rangle_{\mathbb{Z}}$ for all $p \in \mathbb{Z}$. Since $\Gamma$ has rank $k$, the elements $1, \lambda, \ldots, \lambda^{k}$ must be linearly dependent over $\mathbb{Z}$, hence $\lambda$ is an algebraic number over $\mathbb{Q}$. Then $\lambda \in\left\langle B_{A}\right\rangle$. Let $\tau \in B$ be a transcendent element. Then $\lambda \tau$ is transcendent too (may be with an algebraic part). On the other hand, $\lambda$ induces an automorphism $\lambda_{A} \in G L(k, \mathbb{Z})$ of the free abelian group $\left\langle B_{A}\right\rangle$ of rank $k<m$, because the product of algebraic numbers is algebraic. Moreover $\operatorname{det} \lambda_{A}= \pm 1$. That means that the matrix associated to $\lambda$ with respect to $\left\{B_{A}, B_{T}\right\}$ has the form

$$
\left(\begin{array}{cc}
\lambda_{A} & \star \\
0 & \lambda_{T}
\end{array}\right)
$$

where $\lambda_{T} \in G L(m-k, \mathbb{Z})$. In any case, $\operatorname{Aut}_{\Gamma}(\mathbb{R}) \subset \operatorname{Aut}_{\left\langle B_{A}\right\rangle}(\mathbb{R})$ and the same arguments that above apply.

Corollary 6.6. Let $\mathcal{F}$ be a codimension one linear foliation on the torus $T^{m+1}$ with dense leaves. Then

$$
\operatorname{Diff}\left(T^{m+1} / \mathcal{F}\right)=\left(\mathbb{Z}_{2} \times \mathbb{Z} \times \cdots \times \mathbb{Z}\right) \ltimes\left(T^{m+1} / \mathcal{F}\right), \quad r \leq m .
$$

Corollary 6.7 ([2]). Let $\mathcal{F}_{\alpha}$ be an irrational flow on the torus $T^{2}$. Then $\operatorname{Diff}\left(T^{2} / \mathcal{F}_{\alpha}\right)$ is isomorphic to

(1) $\left(Z_{2} \times \mathbb{Z}\right) \ltimes\left(T^{2} / \mathcal{F}_{\alpha}\right)$ if $\alpha$ is a quadratic number;

(2) $\left(Z_{2}\right) \ltimes\left(T^{2} / \mathcal{F}_{\alpha}\right)$ otherwise.

\subsection{Examples.}

Example 6.8. Let $B=1 \cup B_{T}$, with $B_{T}$ a finite set of transcendent elements (chosen like in the proof of Theorem 6.5), such that $B$ is a linearly independent set over $\mathbb{Q}$. We have seen that any $\lambda \neq 0$ inducing an automorphism of $\Gamma=\langle B\rangle$ must be in $\left\langle B_{A}\right\rangle=\mathbb{Z}$ and induce an automorphism of $\left\langle B_{A}\right\rangle=\mathbb{Z}$. Then $\lambda= \pm 1$, so $\operatorname{Aut}_{\Gamma}(\mathbb{R})=\mathbb{Z}_{2}$.

Example 6.9. Let $B=\left\{1,2^{1 / 3}, 2^{2 / 3}\right\}$. Then it can be proved that the integral closure of $\mathbb{Q}\left(2^{2 / 3}\right)$ over $\mathbb{Z}$ is exactly $\Gamma=\langle B\rangle$. The minimal monic polynomial of $2^{2 / 3}$ has a real root and two complex roots, that is $t=1, s=1$. Then $\operatorname{Aut}_{\Gamma}(\mathbb{R})=U(\Gamma)=Z_{2} \times Z$.

Example 6.10. Let $B=\{1, \sqrt{2}, e, \sqrt{2} e\}$. The algebraic part of $\Gamma$ is $\mathbb{Z}[\sqrt{2}]$. Then $B_{A}=\{1, \sqrt{2}\}$ can be completed with $B_{T}=\{e\}$. The unit group of $\mathbb{Z}[\sqrt{2}]$ is $U=\{ \pm 1\} \times\left\{(1+\sqrt{2})^{n}: n \in \mathbb{Z}\right\}$. Then if $\lambda \in U$ we have that $\lambda e$ is an automorhism of $\left\langle B_{T}\right\rangle$ if $\lambda= \pm 1$. Hence $\operatorname{Aut}_{\Gamma}(\mathbb{R})=\mathbb{Z}_{2} \times \mathbb{Z}$. 
As in the latter (quadratic) example, the explicit computation of a basis for the diffeomorphism group would require to solve some algebraic equations (e.g. the Pell-Fermat equation as is done in [17]). We shall prove that excepting quadratic foliations, linear foliations have no transverse diffeomorphisms other that \pm id.

\section{LiNEAR FLOWS ON THE TORUS $T^{n+1}$}

Since a dense flow must have simply connected leaves, by duality we can apply the results of the preceding section to linear flows. The importance of linear flows on the torus comes from the following result of P. Caron and Y. Carrière.

Theorem 7.1 ([1]). Let $M$ be a compact manifold of dimension $n+1$, endowed with a dense $G$-Lie flow. Then $G=\mathbb{R}^{n}, M$ is diffeomorphic to the torus $T^{n+1}$, and the given foliation is conjugate to a linear one.

Let $T^{n+1}=\mathbb{R}^{n+1} / \mathbb{Z}^{n+1}$. We take coordinates $\left(x, y_{1}, \ldots, y_{n}\right)$ in $\mathbb{R} \times \mathbb{R}^{n}$. Let $\omega$ be the closed 1 -form on the torus, with values on $\mathbb{R}^{n}$, given by

$$
\omega=\left(d y_{1}-\alpha_{1} d x, \ldots, d y_{n}-\alpha_{n} d x\right) .
$$

Let $\mathcal{F}_{\alpha}$ be the corresponding flow. The holonomy group is $\Gamma=\left\langle e_{1}, \ldots, e_{n}, \alpha\right\rangle \subset$ $\mathbb{R}^{n}$, where $\alpha=\left(\alpha_{1}, \ldots, \alpha_{n}\right)$ and $e_{1}, \ldots, e_{n}$ is the canonical basis. We shall denote $T_{\alpha}$ the space of leaves.

As we know, the abelian group $\Gamma=\left\langle e_{1}, \ldots, e_{n}, \alpha\right\rangle$ is dense in $\mathbb{R}^{n}$ if and only if the free abelian group $\Gamma^{\star}=\left\langle 1, \alpha_{1}, \ldots, \alpha_{n}\right\rangle \subset \mathbb{R}$, corresponding to the orthogonal foliation, has rank $n+1$. Then the group $\operatorname{Aut}_{\Gamma}\left(\mathbb{R}^{n}\right)$ is isomorphic to $\operatorname{Aut}_{\Gamma^{*}}(\mathbb{R})$. In fact, each automorphism in $\operatorname{Aut}_{\Gamma}\left(\mathbb{R}^{n}\right)$ corresponds to an integer matrix $A$ such that $A \cdot \alpha=\alpha$. So we must compute the stabilizer of $\alpha$ for the action of $G L(n+1, \mathbb{Z})$. This is exactly what we did in Proposition 6.2, applied to the groups $\Gamma_{\alpha}^{\star}$ and $\Gamma_{\beta}^{\star}$.

Corollary 7.2. The diffeomorphism group $\operatorname{Diff}\left(T^{n+1} / \mathcal{F}\right)$ is isomorphic to $\left(\mathbb{Z}_{2} \times \mathbb{Z} \times \stackrel{r}{ } . . \times \mathbb{Z}\right) \ltimes\left(T^{n+1} / \mathcal{F}\right)$. Moreover $r \leq n$.

7.1. Classification. Again we can classify the spaces of leaves, this time for linear flows on $T^{n+1}$.

Proposition 7.3. Let $\mathcal{F}_{\alpha}, \mathcal{F}_{\beta}$ be two dense linear flows on the torus $T^{n+1}$, respectively associated to the vectors $\alpha=\left(\alpha_{1}, \ldots, \alpha_{n}\right), \beta=\left(\beta_{1}, \ldots, \beta_{n}\right)$. Then their spaces of leaves $T_{\alpha}, T_{\beta}$ are diffeomorphic if and only if $\alpha$ and $\beta$ are $G L(n+1, \mathbb{Z})$ related.

Proof. We know that the existence of a diffeomorphism is equivalent to that of some linear automorphism $\varphi=\left(f_{i j}\right)$ of $\mathbb{R}^{n}$ such that $\varphi\left(\Gamma_{\alpha}\right)=\Gamma_{\beta}$. Since $\varphi\left(e_{j}\right) \in \Gamma_{\beta}$, we have

$$
\left(f_{1 j}, \ldots, f_{n j}\right)=\left(c_{1 j}+b_{j} \beta_{1}, \ldots, c_{n j}+b_{j} \beta_{n}\right), \quad 1 \leq j \leq n,
$$


with $c_{i j}, b_{j} \in \mathbb{Z}$. Since $\varphi(\alpha) \in \Gamma_{\beta}$, we also have

$$
f_{i 1} \alpha_{1}+\cdots+f_{i n} \alpha_{n}=c_{i}+b_{n+1} \beta_{i}, \quad 1 \leq i \leq n,
$$

with $c_{i}, b_{n+1} \in \mathbb{Z}$. From equations (11) and (2) we obtain

$$
\beta_{i}=\frac{c_{i}-c_{i 1} \alpha_{1}-\cdots-c_{i n} \alpha_{n}}{-b_{n+1}+b_{1} \alpha_{1}+\cdots+b_{n} \alpha_{n}}, \quad 1 \leq i \leq n
$$

that is $\beta=A \cdot \alpha$ for

$$
A=\left(\begin{array}{cccc}
-b_{n+1} & c_{1} & \cdots & c_{n} \\
b_{1} & -c_{11} & \cdots & -c_{n 1} \\
\vdots & \vdots & & \vdots \\
b_{n} & -c_{1 n} & \cdots & -c_{n n}
\end{array}\right) \in G L(n+1, \mathbb{Z})
$$

When $n=1$ the latter result was proven in [2] (see also Corollary 6.4).

\section{Transcendent and Quadratic FOliations}

Our next generalization of the results of [2] involves arbitrary linear foliations of dimension $m \geq 1$ and codimension $n \geq 1$ on the torus $T^{m+n}$. Such a foliation is determined by some linear subspace $V \subset \mathbb{R}^{m+n}$ of dimension $m$. By taking dual coordinates, we have a closed 1-form $\omega=\left(\omega_{1}, \ldots, \omega_{n}\right)$ with values in $\mathbb{R}^{n}$ which defines the foliation.

8.1. Transcendent foliations. Let us say that the foliation is transcendent if the invariant subspace $V$ admits a basis

$$
\begin{aligned}
v_{1} & =\left(\alpha_{1}^{1}, \ldots, \alpha_{m}^{1}, \beta_{1}^{1}, \ldots, \beta_{n}^{1}\right) \\
& \vdots \\
v_{m} & =\left(\alpha_{1}^{m}, \ldots, \alpha_{m}^{m}, \beta_{1}^{m}, \ldots, \beta_{n}^{m}\right)
\end{aligned}
$$

such that all the coordinates $\alpha_{i}^{j}, \beta_{i}^{j}$ are algebraically independent over $\mathbb{Q}$. The basis $\left\{v_{1}, \ldots, v_{m}\right\}$ will be called a transcendence basis. Notice that a transcendent foliation has dense leaves, because, accordingly to what we have seen in the preceding sections, the flow generated by any of the $v_{i}$ is dense.

Transcendent foliations appeared in B. Herrera's thesis 10. Our definition is easily seen to be equivalent to the original one (where the last coordinates of the $v_{i}$ 's are supposed to be 1 ).

Theorem 8.1 ([10]). The only transverse diffeomorphisms of a transcendent foliation are \pm id.

We shall generalize this result by dualizing the definition above.

Lemma 8.2. The subspace $V \subset \mathbb{R}^{m+n}$ generates a transcendent foliation if and only if its orthogonal subspace $W=V^{\perp}$ generates a transcendent foliation. 
Proof. If $n=1$, let us take a transcendence basis of $V$, and the vector

$$
w=v_{1} \wedge \cdots \wedge v_{m}=\operatorname{det}\left(\begin{array}{cccc}
e_{1} & \ldots & e_{m} & e_{m+1} \\
\alpha_{1}^{1} & \ldots & \alpha_{m}^{1} & \beta_{1}^{1} \\
\vdots & & \vdots & \vdots \\
\alpha_{1}^{m} & \ldots & \alpha_{m}^{m} & \beta_{1}^{m}
\end{array}\right),
$$

which is a basis of $W$, and whose coordinates remain algebraically independent over $\mathbb{Q}$.

When $V$ has codimension $n \geq 2$, we can complete any transcendence basis $\left\{v_{1}, \ldots, v_{m}\right\}$ of $V$ to a transcendence basis $\left\{v_{1}, \ldots, v_{m}, u_{1}, \ldots, u_{n}\right\}$ of $\mathbb{R}^{m+n}$, because the set of real numbers which are algebraically dependent over any extension $\mathbb{Q}\left(t_{1}, \ldots, t_{r}\right), t_{1}, \ldots, t_{r} \in \mathbb{R}$, is a countable set. Let us consider the vectors

$$
w_{j}=v_{1} \wedge \cdots \wedge v_{m} \wedge u_{1} \wedge \cdots \wedge \widehat{u_{j}} \wedge \cdots \wedge u_{n}, \quad 1 \leq j \leq n .
$$

Then $\left\{w_{1}, \ldots, w_{n}\right\}$ is a transcendence basis of $W$.

Corollary 8.3. The foliation $\mathcal{F}$ is transcendent if and only if the coordinates of some closed form $\omega=\left(\omega_{1}, \ldots, \omega_{n}\right)$ defining it are a set of algebraically independent numbers over $\mathbb{Q}$.

Proof. The coordinates of $\omega$ with respect to the dual basis

$$
d x_{1}, \ldots, d x_{m}, d y_{1}, \ldots, d y_{n}
$$

just correspond to the orthogonal subspace $W$.

Proposition 8.4. The foliation is transcendent if and only if it can be defined by 1-forms

$$
\omega_{j}=d y_{j}+\beta_{j}^{1} d x_{1}+\cdots+\beta_{j}^{m} d x_{m}, \quad 1 \leq j \leq n,
$$

such that the coefficients $\left\{\beta_{i}^{j}\right\}, 1 \leq i \leq m, 1 \leq j \leq n$, are algebraically independent.

Proof. Since the foliation is transcendent, we have $\operatorname{det}\left(\alpha_{i}^{j}\right) \neq 0$ for the coefficients of the transverse part of the forms defining the foliation. The corresponding change of basis respects the algebraic independence of the tangential coefficients.

8.2. Quadratic foliations. The considerations of the preceding subsection suggest the following definition.

Definition 8.5. Let us say that a codimension $n$ linear foliation $\mathcal{F}$ on the torus $T^{m+n}$ is non-quadratic whenever it can be defined by a closed $\mathbb{R}^{n}$ valued 1 -form $\omega=\left(\omega_{1}, \ldots, \omega_{n}\right)$, where

$$
\omega_{j}=d y_{j}+\beta_{j}^{1} d x_{1}+\cdots+\beta_{j}^{m} d x_{m}, \quad 1 \leq j \leq n,
$$

such that any polynomial with $m n$ variables and rational coefficients that annihilates the family of coefficients $\left\{\beta_{j}^{i}\right\}$ has its degree greater than 2. 
Obviously a transcendent foliation is not quadratic.

Example 8.6. The foliation on $T^{3}$ defined by the 2-subspace $V \subset \mathbb{R}^{3}$ with basis $v_{1}=\left(1,0,2^{1 / 3}\right), v_{2}=\left(0,1,3^{1 / 3}\right)$ is neither transcendent nor quadratic.

Theorem 8.7. Let $\mathcal{F}$ be a non-quadratic linear foliation. Then $\operatorname{Aut}_{\Gamma}\left(\mathbb{R}^{n}\right)=$ $\{ \pm \mathrm{id}\}$ and $\operatorname{Diff}\left(T^{m+n} / \mathcal{F}\right)=\mathbb{Z}_{2} \ltimes\left(T^{m+n} / \mathcal{F}\right)$.

Proof. The holonomy group is $\Gamma=\left\langle e_{1}, \ldots, e_{n}, \beta_{1}, \ldots, \beta_{m},\right\rangle \subset \mathbb{R}^{n}$, where $\beta_{k}=\left(\beta_{1}^{k}, \ldots, \beta_{n}^{k}\right)$. In the same way as we $\operatorname{did}$ in Proposition 7.3 , one can prove that the matrix $\left(f_{i j}\right)$ associated to a linear automorphism $\varphi \in$ $\operatorname{Aut}_{\Gamma}\left(\mathbb{R}^{n}\right)$ must verify

$$
f_{i j}=c_{i j}+b_{1}^{j} \beta_{i}^{1}+\cdots+b_{m}^{j} \beta_{i}^{m}, \quad 1 \leq i, j \leq n,
$$

with $c_{i j}, b_{i}^{j} \in \mathbb{Z}$ because $\varphi\left(e_{j}\right) \in \Gamma, 1 \leq j \leq n$. From the conditions $\varphi\left(\beta_{k}\right) \in$ $\Gamma, 1 \leq k \leq m$, we deduce relations of algebraic dependence over $\mathbb{Q}$ for the family $\left\{\beta_{k}^{j}\right\}$, that is

$$
f_{i 1} \beta_{1}^{k}+\cdots+f_{i n} \beta_{n}^{k} \in\left\langle 1, \beta_{i}^{j}\right\rangle_{\mathbb{Z}}, \quad 1 \leq i \leq k, 1 \leq k \leq m .
$$

These relations are given by a rational polynomial on $m n$ variables, having degree not greater than 2 , excepting when $c_{i}^{j}=0,1 \leq i \leq m, 1 \leq j \leq n$, and $m_{i j}=0$ when $i \neq j$. Hence the matrix is diagonal with the same number $m \in \mathbb{Z}$ in all entries. Since $\varphi$ induces an automorphism of $\Gamma$, we deduce analogous conditions for the inverse matrix, so $m= \pm 1$ and $\varphi= \pm$ id.

For $n=1$ this is again Donato-Iglesias' result cited in Corollary 6.7 For transcendent foliations it has been proved by B. Herrera in [10].

8.3. Classification. The proof of the following theorem is analogous to that of Proposition 7.3 and we shall omit it.

Theorem 8.8. Let $\mathcal{F}, \mathcal{F}^{\prime}$ be two non-quadratic linear foliations on $T^{m+n}$ associated to the coefficients $\beta_{1}, \ldots, \beta_{m} \in \mathbb{R}^{n}$ and $\gamma_{1}, \ldots, \gamma_{m} \in \mathbb{R}^{n}$ respectively. Then the spaces of leaves $T^{m+n} / \mathcal{F}$ and $T^{m+n} / \mathcal{F}^{\prime}$ are diffeomorphic (in the diffeological sense) if and only if

$$
\gamma=(A+\beta B)^{-1}(C+\beta D)
$$

for integer matrices $A \in \mathcal{M}_{n \times n}(\mathbb{Z}), B \in \mathcal{M}_{m \times m}(\mathbb{Z}), C \in \mathcal{M}_{n \times m}(\mathbb{Z})$ and $D \in \mathcal{M}_{m \times n}(\mathbb{Z})$, where $\beta, \gamma$ are the matrices whose columns are the given coefficients of the foliation.

This could be stated in terms of the action of $G L(m+n, \mathbb{Z})$ on the grasmannian $G_{n}^{m+n}$. For $m=1, n=1$ we reobtain Corollary 6.4.

\section{REFERENCES}

[1] P. Caron and Y. Carrière. Flots transversalement de lie $r^{n}$, flots transversalement de lie minimaux. Comptes Rendus Acad. Sci. Paris, 291:477-478, 1980.

[2] P. Donato and P. Iglesias. Exemples de groupes difféologiques: flots irrationels sur le tore. Comptes Rendus Acad. Sci. Paris, 301(4):127-130, 1985. 
[3] A. El Kacimi and A. Tihami. Cohomologie bigraduée de certains feuilletages. Bull. Soc. Math. Bélgique, 38(2):144-157, 1986.

[4] A. El Kacimi-Alaoui and A. Tihami. Erratum á: Cohomologie bigraduée de certains feuilletages. Bull. Soc. Math. Bélgique, 379(B 39):144-157, 1987.

[5] E. Fédida. Sur les feuilletages de lie. Comptes Rendus Acad. Sci. Paris, 272:999-1001, 1971.

[6] E. Fédida. Feuilletages de Lie, feuilletages du plan. PhD thesis, Université de Strasbourg, 1973.

[7] G. Hector. Géométrie et topologie des espaces difféologiques. In X. Masa, E. MaciasVirgós, and J.A. Álvarez-López, editors, Analysis and geometry in foliated manifolds. Proceedings VII Int. Coll. Diff. Geom., Santiago de Compostela, Spain, July 26-30 1994, pages 55-80. World Scientific, Singapore, 1995.

[8] G. Hector and E. Macias. Diffeological groups. In Ignacio Bajo and et al., editors, Recent advances in Lie theory. Selected contributions to the 1st colloquium on Lie theory and applications, Vigo, Spain, July 17-22, 2000, volume 25 of Res. Expo. Math., pages 247-260. Lemgo: Heldermann Verlag, 2002.

[9] B. Herrera, M. Llabrés, and A. Reventós. Transverse structure of lie foliations. J. Math. Soc. Japan, 48(4):769-795, 1996.

[10] B. Herrera Gómez. Sobre la estructura transversa de las foliaciones de Lie. PhD thesis, Univ. Autónoma de Barcelona, 1994.

[11] P. Iglesias. Fibrations difféologiques et homotopie. PhD thesis, Université de Provence, Marseille, France, 1985.

[12] P. Iglesias and G. Lachaud. Espaces différentiables singuliers et corps de nombres algébriques. Ann. Inst. Fourier Grenoble, 40(3):723-737, 1990.

[13] P. Iglesias-Zemmour. Diffeology. eprint http://math.huji.ac.il/ piz/Site/The\%20Book/The\%20Book.html, 2007.

[14] S. Lang. Algebraic number theory. Springer-Verlag, 1986.

[15] E. Macias-Virgós. Homotopy groups in lie foliations. Trans. Am. Math. Soc., 344(2):701-711, 1994.

[16] P. Molino. Riemannian foliations. Birkhauser, 1988.

[17] A. Sotelo Armesto. El grupo de difeomorfismos del espacio de hojas de una foliación de Lie desde un punto de vista difeológico, volume 99 of Publ. Dpto. Geom. y Top. Univ. Santiago de Compostela, 2003.

[18] J.-M. Souriau. Groupes différentiels, pages 91-128. Springer, 1980.

[19] I. Stewart and D. Tall. Algebraic number theory. Chapman and Hall, 1987.

Institut Camille Jordan, Université Claude Bernard Lyon 1, 69622-Villeurbanne (FRANCE)

E-mail address: gilb.hector@gmail.com

Dpto. Xeometria e Topoloxia, Facultade de Matemáticas, Universidade de Santiago de Compostela, 15782-Santiago de Compostela (Spain)

E-mail address: quique.macias@usc.es

Dpto. Xeometria e Topoloxia, Facultade de Matemáticas, Universidade de Santiago de Compostela, 15782-Santiago de Compostela (Spain) 